\title{
ELECTRON TRAJECTORIES IN A REALISTIC GYROTRON RESONATOR
}

\author{
OLGERTS DUMBRAJS ${ }^{1}$ AND ANDREJS REINFELDS ${ }^{2}$ \\ ${ }^{1}$ Department of Engineering Physics and Mathematics, \\ Helsinki University of Technology, Espoo, FIN-02150 Finland; \\ E-mail: olgierd.dumbrajs@hut.fi \\ ${ }^{2}$ Institute of Mathematics and University of Latvia; \\ Akademijas laukums 1, Riga, LV-1524 Latvia; \\ E-mail: reinf@latnet.lv
}

\begin{abstract}
Gyrotron is a special tube generating powerful radiowaves in the millimeter wave range. Gyrotrons are mainly used to heat nuclear fusion plasma, in order to induce controlled thermonuclear reactions on earth. In addition, they have found a wide utility in radars and the high-temperature processing of materials. Differential equations describing gyrotron operation are analyzed from the mathematical point of view. Phase portraits of electron trajectories in realistic resonators are determined.
\end{abstract}

\section{GYROTRON EQUATIONS}

The simplest equation which describes the electron motion in a gyrotron resonator can be written as follows:

$$
\frac{d p}{d \zeta}+i\left(\Delta+|p|^{2}-1\right) p=i f(\zeta) F
$$

with the initial condition $p\left(\zeta_{0}\right)=\left|p_{0}\right| \exp \left(i \theta_{0}\right)$, where $0 \leq \theta_{0}<2 \pi$.

Here $p$ is the dimensionless transverse momentum of the electron, $\zeta$ is the dimensionless coordinate, $\Delta$ is the frequency mismatch, and $F$ is the beamhigh-frequency field coupling. Equation (1) represents the so-called coldcavity approximation in the gyrotron theory when the high-frequency field in a gyrotron resonator $f(\zeta)$ depends only on the geometry of the resonator, but not on the electron motion, i.e., $f$ does not depend on $p$. In this case the 
high-frequency field in a cavity is usually represented by a Gaussian

$$
f(\zeta)=\exp \left[-\left(\frac{2 \zeta}{\mu}-\sqrt{3}\right)^{2}\right]
$$

where $\mu$ is the dimensionless length of the resonator.

In [1] equation (1) was examined in detail from the mathematical point of view. In particular electron trajectories were classified and the asymtotical equivalence of (1) and the corresponding unforced equation $(f(\zeta) \equiv 0)$

$$
\frac{d q}{d \zeta}+i\left(\Delta+|q|^{2}-1\right) q=0, \quad q\left(\zeta_{0}\right)=q_{0} .
$$

was proved in the case when the function $f(\zeta)$ decays fast enough for large $\zeta$ : faster than $1 / \zeta^{2+\epsilon}$. In such a case each solution of (1) corresponds to a solution of (3) with changed initial data.

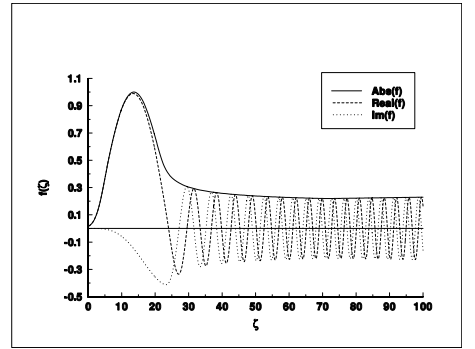

Figure 1. The graphs of $|f|, \Re(f)$ and $\Im(f)$

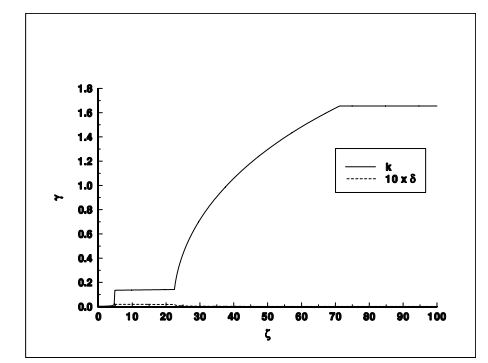

Figure 2. The graph of $\gamma$

It should be emphasized that the high-frequency field in the cavity represented by a Gaussian in (2) is an approximation which is valid in resonators with very high quality factors. In realistic cases even in the cold-cavity approximation one should determine $f(\zeta)$ for each specific resonator geometry. For this purpose the following second-order differential equation has to be solved:

$$
\frac{d^{2} f}{d \zeta^{2}}+\gamma(\zeta)^{2} f=0
$$

with the boundary conditions

$$
\begin{gathered}
\frac{d f}{d \zeta}=i \gamma(\zeta) f, \quad \zeta=0 \\
\frac{d f}{d \zeta}=-i \gamma(\zeta) f, \quad \zeta=\zeta_{\text {out }}
\end{gathered}
$$


Here $\gamma(\zeta)=k(\zeta)+i \delta(\zeta)$. In Fig. 1 we show a typical example of $f(\zeta)$. The corresponding function $\gamma(\zeta)$ is shown in Fig. 2. As is evident from these figures, for $\zeta \geq 70$ we can approximate $f(\zeta)$ as a wave moving in $+\zeta$ direction: $f(\zeta) \sim e^{-i \gamma \zeta}$. Moreover, since $k \gg \delta$, we can write $f(\zeta) \sim e^{-i k \zeta}$.

\section{MODIFIED EQUATIONS}

Let us consider the equation

$$
\frac{d \tilde{p}}{d \zeta}+i\left(\Delta+|\tilde{p}|^{2}-1\right) \tilde{p}=i f(\zeta) e^{-i k \zeta} F, \quad \tilde{p}(0)=\tilde{p}_{0},
$$

where $f(\zeta) \rightarrow 1$ if $\zeta \rightarrow+\infty$, and $F>0$. We denote the solution to this equation by $\tilde{p}\left(\zeta, \tilde{p}_{0}\right)$. We wish to find a simpler equation such that each solution of (5) corresponds to a solution of the simpler equation $p\left(\zeta, p_{0}\right)$ (in general with different initial conditions) and the difference

$$
\left|\tilde{p}\left(\zeta, \tilde{p}_{0}\right)-p\left(\zeta, p_{0}\right)\right| \rightarrow 0,
$$

if $\zeta \rightarrow+\infty$.

A detailed analysis proves that the structure of the solutions of the equation

$$
\frac{d p}{d \zeta}+i\left(\Delta+|p|^{2}-1\right) p=0
$$

is essentialy different from the structure of the solutions of (5). For this reason it is expedient to compare (5) with the equation

$$
\frac{d p}{d \zeta}+i\left(\Delta+|p|^{2}-1\right) p=i e^{-i k \zeta} F, \quad p(0)=p_{0} .
$$

Let us introduce the new function $p=e^{-i k \zeta} q$. After the transformation we obtain the equations

$$
\frac{d \tilde{q}}{d \zeta}+i\left(\Delta+|\tilde{q}|^{2}-1-k\right) \tilde{q}=i f(\zeta) F
$$

and

$$
\frac{d q}{d \zeta}+i\left(\Delta+|q|^{2}-1-k\right) q=i F
$$

If (7) and (8) are asymptotically equivalent, i.e., if the homeomorphism exists $H: C \rightarrow C$, such that

$$
\left|\tilde{q}\left(\zeta, p_{0}\right)-q\left(\zeta, H\left(p_{0}\right)\right)\right| \rightarrow 0,
$$


for $\zeta \rightarrow+\infty$, then going over to (5) and (6), we obtained

$$
\begin{array}{r}
\left|\tilde{p}\left(\zeta, p_{0}\right)-p\left(\zeta, H\left(p_{0}\right)\right)\right|=\left|e^{-i k \zeta} \tilde{q}\left(\zeta, p_{0}\right)-e^{-i k \zeta} q\left(\zeta, H\left(p_{0}\right)\right)\right| \\
=\left|\tilde{q}\left(\zeta, p_{0}\right)-q\left(\zeta, H\left(p_{0}\right)\right)\right| \rightarrow 0,
\end{array}
$$

for $\zeta \rightarrow+\infty$. From this it follows that (5) and (6) are asymtotically equivalent, if and only if (7) and (8) are asymptotically equivalent.

Another important property of (8) should be mentioned. It is an autonomous equation, i.e., its right-hand side does not depend on $\zeta$. This makes it possible to obtain illustrative trajectories in the phase space.

\section{QUALITATIVE ANALYSIS OF EQUATION (8)}

In the polar coordinates where $q=r e^{i \theta}$, we obtain

$$
\left\{\begin{array}{lll}
\frac{d r}{d \zeta}=F \sin \theta, & r\left(\zeta_{0}\right)=r_{0} \\
r \frac{d \theta}{d \zeta}=\left(1-\Delta-r^{2}+k\right) r+F \cos \theta, & \theta\left(\zeta_{0}\right)=\theta_{0} .
\end{array}\right.
$$

The differential equation for trajectories is

$$
\left(F \cos \theta+\left(1-\Delta-r^{2}+k\right) r\right) d r=F r \sin \theta d \theta .
$$

Introducing the new auxiliary function $z=\cos \theta$, we obtain

$$
\left(F z+\left(1-\Delta-r^{2}+k\right) r\right) d r+F r d z=0 .
$$

By transforming

$$
4 F d(r z)+d\left(2(1-\Delta+k) r^{2}-r^{4}\right)=0,
$$

integrating

$$
4 F r z+2(1-\Delta+k) r^{2}-r^{4}=4 F r_{0} z_{0}+2(1-\Delta+k) r_{0}^{2}-r_{0}^{4},
$$

and returning to the old variables, we obtain

$$
\begin{aligned}
4 F r \cos \theta+2(1-\Delta+k) r^{2}-r^{4}= & 4 F r_{0} \cos \theta_{0} \\
& +2(1-\Delta+k) r_{0}^{2}-r_{0}^{4} .
\end{aligned}
$$

In the Cartesian coordinates we obtain a set of fourth order algebraic curves

$$
\begin{array}{r}
4 F x+2(1-\Delta+k)\left(x^{2}+y^{2}\right)-\left(x^{2}+y^{2}\right)^{2}=4 F x_{0} \\
+2(1-\Delta+k)\left(x_{0}^{2}+y_{0}^{2}\right)-\left(x_{0}^{2}+y_{0}^{2}\right)^{2}
\end{array}
$$


From this it follows that any trajectory of (8) is bounded, and that no spirallike trajectories exist. In principle we could integrate the system of equations (9) using the Jacobi elliptic functions.

Instead we will use the qualitative theory of differential equations to study the phase portrait of (8). Separating the real and imaginary parts, we obtain the following system of equations:

$$
\left\{\begin{array}{l}
\frac{d x}{d \zeta}=-(1-\Delta+k) y+y\left(x^{2}+y^{2}\right) \\
\frac{d y}{d \zeta}=(1-\Delta+k) x-x\left(x^{2}+y^{2}\right)+F
\end{array}\right.
$$

First, we determine the number and types of stationary points of the system (13). Stationary points of this system are $(a, 0)$, where $a$ are roots of the cubic equation

$$
x^{3}-(1-\Delta+k) x-F=0 .
$$

We consider the case when $\lambda=1-\Delta+k>0$. The cubic equation has at least one positive root $a_{1}>\sqrt{\lambda}$. The following cases are possible:

1. if $3 \sqrt{3} F>2 \lambda \sqrt{\lambda}$, there is one positive root $2 \sqrt{\frac{\lambda}{3}}<a_{1}<\sqrt[3]{4 F}$;

2. if $3 \sqrt{3} F=2 \lambda \sqrt{\lambda}$, there is one positive root $a_{1}=2 \sqrt{\frac{\lambda}{3}}$ and two negative coinciding roots $a_{2,3}=-\sqrt{\frac{\lambda}{3}}$;

3. if $3 \sqrt{3} F<2 \lambda \sqrt{\lambda}$, there is one positive and two different negative roots, where $a_{1}>\sqrt{\lambda},-\sqrt{\lambda}<a_{3}<-\sqrt{\frac{\lambda}{3}}$ and $-\sqrt{\frac{\lambda}{3}}<a_{2}<0$.

We now determine the type of stationary points corresponding to the linear approximation. We evalute the Jacobi matrix of the right-hand side of the system at stationary points. We find that $\operatorname{Tr} J=0$ and $\left.\operatorname{det} J\right|_{x=a, y=0}=$ $\lambda^{2}-4 \lambda^{2}+3 a^{4}=\left(\lambda-a^{2}\right)\left(\lambda-3 a^{2}\right)$. Hence, the stationary point of the linear approximation is a saddle, if $\operatorname{det} J<0$, a center, if $\operatorname{det} J>0$, or a complex point, if $\operatorname{det} J=0$. In our case: if $\sqrt{\frac{\lambda}{3}}<|a|<\sqrt{\lambda}$, then $\operatorname{det} J<0$; if $|a|<\sqrt{\frac{\lambda}{3}}$, or $|a|>\sqrt{\lambda}$, then $\operatorname{det} J>0$; if $|a|=\sqrt{\frac{\lambda}{3}}$, or $|a|=\sqrt{\lambda}$, then $\operatorname{det} J=0$. Thus, we have:

1. The case of the positive root. Here $a_{1}>\sqrt{\lambda}$ and $\left.\operatorname{det} J\right|_{x=a_{1}, y=0}>0$.

2. The case of the double negative root. Here $a_{2}=a_{3}=-\sqrt{\frac{\lambda}{3}}$ and $\left.\operatorname{det} J\right|_{x=a_{2,3}, y=0}=0$

3. The case of two different negative roots. Here $-\sqrt{\lambda}<a_{3}<-\sqrt{\frac{\lambda}{3}}$ and $-\sqrt{\frac{\lambda}{3}}<a_{2}<0$. It follows that $\left.\operatorname{det} J\right|_{x=a_{3}, y=0}<0$ un $\left.\operatorname{det} J\right|_{x=a_{2}, y=0}>0$.

Since the trajectories of (10) are fourth order algebraic curves, then for $\operatorname{det} J>$ 0 we find that the corresponding stationary point is a center. 
There three types of phase portraits for (13). The type depends on the sign of the expression

$$
3 \sqrt{3} F-2 \lambda \sqrt{\lambda} .
$$

1. If $3 \sqrt{3} F-2 \lambda \sqrt{\lambda}>0$, then the equation has one stationary point $\left(a_{1}, 0\right)$ which is a center. Other trajectories are closed curves (homeomorphic to a unit circle). The phase point rotates along the trajectory clockwise. The corresponding solutions are periodic functions where periods are changing and are continuously dependent on initial conditions. If the trajectory approaches the stationary point, then its period approaches the value

$$
T=\frac{2 \pi}{\sqrt{\left(a_{1}^{2}-\lambda\right)\left(3 a_{1}^{2}-\lambda\right)}}
$$

and if $x_{0}^{2}+y_{0}^{2} \rightarrow+\infty$, then $T\left(x_{0}^{2}+y_{0}^{2}\right) \rightarrow 2 \pi$.

2. If $3 \sqrt{3} F-2 \lambda \sqrt{\lambda}=0$, then the phase portrait is similar to the previous case. One new stationary point is born $\left(a_{2,3}, 0\right)$ on one closed trajectory, and instead of the corresponding closed trajectory two trajectories appear: the stationary point $\left(a_{2,3}, 0\right)$ and a loop which originates and ends at this stationary point. The periods of the closed trajectories approach $+\infty$, if the origin approaches the loop. The periods approache

$$
T=\frac{2 \pi}{\lambda},
$$

if the origin approaches $\left(a_{1}, 0\right)$.

Note. Let us prove that in the two cases disscussed above the direction of rotation of the phase point is clockwise. In (8) we go over to a new variable by means of the formula

$$
q=r e^{i \theta}+a_{1},
$$

where $a_{1}$ is the root of the corresponding cubic equation. After transformations we obtain the following system of equations:

$$
\left\{\begin{array}{l}
\frac{d r}{d \zeta}=-a_{1}\left(r^{2}+2 a_{1} r \cos \theta\right) \sin \theta, \\
\frac{d \theta}{d \zeta}=-r^{2}-3 a_{1} r \cos \theta-a_{1}^{2}\left(1+2 \cos ^{2} \theta\right)+1-\Delta+k .
\end{array}\right.
$$

The right-hand side of the second equation is a quadratic trinomial in $r$. Let us determine the sign of the discriminant

$$
\begin{gathered}
D=9 a_{1}^{2} \cos ^{2} \theta+4\left(1-\Delta+k-a_{1}^{2}\left(1+2 \cos ^{2} \theta\right)\right)=4\left(1-\Delta+k-a_{1}^{2}\right)+a_{1}^{2} \cos ^{2} \theta \\
=-\frac{4 F}{a_{1}}+a_{1}^{2} \cos ^{2} \theta=\frac{a_{1}^{3} \cos ^{2} \theta-4 F}{a_{1}}<\frac{4 F \cos ^{2} \theta-4 F}{a_{1}} \leq 0 .
\end{gathered}
$$

It follows that $\frac{d \theta}{d \zeta}<0$ which means that the phase point rotates clockwise.

3. If $3 \sqrt{3} F-2 \lambda \sqrt{\lambda}<0$, then the phase portrait is somewhat more complicated. The stationary point $\left(a_{2}, 0\right)$ splits into two stationary points, one of which $\left(a_{3}, 0\right)$ is a saddle whose two separatrices form a loop which encloses the second stationary point $\left(a_{3}, 0\right)$ which is a center. Hence, there exist the following trajectories: 
i - three stationary points: one saddle and two centers;

ii - two separatrices of the saddle where the phase points rotate in opposite directions;

iii - closed trajectories whose periods vary from 0 to $+\infty$ approaching loops of trajectories and the phase point rotates both clockwise and counterclock wise.

It is possible to find the equation for separatrices. It turns out that the separatrices form the Pascal limacon.

Note. Determination of the equation for separatrices. We use the trajectory equation and without loosing generality assume that $y_{0}=0$. Then

$$
\left(x^{2}+y^{2}\right)^{2}-2(1-\Delta+k)\left(x^{2}+y^{2}\right)-4 F x=x_{0}^{4}-2(1-\Delta+k) x_{0}^{2}-4 F x_{0}
$$

Introducing the new variable $x=u+a_{3}$, we obtain

$\left(\left(u+a_{3}\right)^{2}+y^{2}\right)^{2}-2(1-\Delta+k)\left(\left(u+a_{3}\right)^{2}+y^{2}\right)-4 F\left(u+a_{3}\right)=a_{3}^{4}-2(1-\Delta+k) a_{3}^{2}-4 F a_{3}$.

After some transformations we find

$$
\left(u^{2}+y^{2}+2 a_{3} u\right)^{2}=2\left(1-\Delta+k-a_{3}^{2}\right)\left(u^{2}+y^{2}\right)+4 u\left(F+(1-\Delta+k) a_{3}-a_{3}^{3}\right) .
$$

Since $a_{3}$ is the root of the corresponding cubic equation, we obtain the equation for the Pascal limacon

$$
\left(u^{2}+y^{2}+2 a_{3} u\right)^{2}=2\left(1-\Delta+k-a_{3}^{2}\right)\left(u^{2}+y^{2}\right) .
$$

\section{SOLUTIONS OF EQUATION (6)}

Taking into account what has been said above, we can state that any solution of (6) can be repesented in the form

$$
p\left(\zeta, p_{0}\right)=e^{-i k \zeta} q\left(\zeta, p_{0}\right),
$$

which is a product of two different periodic functions (except separatrices). This means that the corresponding solution is a periodic function with the minimum period $n k$, where $n \in N$, or an almost periodic function. By projecting the integral line of (6) onto the phase plane, we find a complicated behavior which reminds chaos. All we can say that

$$
\left|p\left(\zeta, p_{0}\right)\right|=\left|q\left(\zeta, p_{0}\right)\right|
$$

which means that the projection of the integral line is located in a ring which is determined by the modulus of the solution of (8).

\section{REFERENCES}

[1] O. Dumbrajs, R. Meyer-Spasche and A. Reinfelds, Analysis of electron trajectories in a gyrotron resonator, IEEE Trans. Plasma Science 26 (1998), No. 3, P. 846-853. 